0038-1098(94)E0228-4

\title{
THE d-d EXCHANGE INTERACTION IN THE DILUTED MAGNETIC SEMICONDUCTOR $\mathrm{Cd}_{1-x} \mathrm{Fe}_{\mathbf{x}} \mathrm{S}$
}

\author{
A.Twardowski \\ Institute of Experimental Physics, Warsaw University, 00681 Warsaw, Poland \\ Y.F.Chen \\ Department of Physics, National Taiwen University, Taipel, Taiwan10617. R.O.C. \\ W.C.Chou \\ Chung Yuan Christian University, Chung L, Taiwan 32023, R.O.C. \\ M.Demianiuk \\ Institute of Technical Physics, W.A.T., 00908 Warsaw, Poland
}

(Received by M.Grynberg 25 February 1994)

(Accepted 10 March 1994)

\begin{abstract}
We have measured the magnetic susceptibility of $\mathrm{Cd}_{1-x} \mathrm{Fe}_{x} \mathrm{~S}(x<0.09)$ and found a typical Curie-Weiss behaviour above 100K. Basing on the high temperature expansion series developed for Fe-based diluted magnetic semiconductors, we estimate the nearest neighbour exchange coupling constant $\mathrm{J}_{\mathrm{N}}=-20 \mathrm{~K}$.
\end{abstract}

Diluted magnetic semiconductors (DMS) are materials based on classical semiconductors, such as CdTe, ZnS or InAs, for which a controlled fraction of nonmagnetic cations is substituted by magnetic ions such as $\mathrm{Mn}$, Co or Fe [1]. From magnetic point of view DMS are systems of localized magnetic moments (associated with d-electrons of magnetic ions), randomly distributed over a nonmagnetic host lattice and coupled by the d-d exchange interaction. The situation for $\mathrm{Mn}$ - and $\mathrm{Co}$ DMS is relatively simple, since for both $\mathrm{Mn}^{+4}$ and $\mathrm{Co}^{+4}$ ions, the ion ground state is an orbital singlet, which results in a spin-onty magnetic moment. The case of Fe-DMS is different. The cubic crystal field splits the ${ }^{5} \mathrm{D}$ ground term into an orbital doublet ${ }^{5} \mathrm{E}$ and an orbital triplet ${ }^{5} \mathrm{~T}_{2}$ (located about $4000 \mathrm{~K}$ above ${ }^{5} \mathrm{E}$ ) $[2,3]$. Spinorbit interaction yields further splittings, in particular the ${ }^{3} E$ state is split into a singlet $A_{1}$, a triplet $T_{2}$ a doublet $E_{1}$ a triplet $T_{2}$ and a singlet $A_{2}[2,3]$. The separation between these five states is roughly $15-20 \mathrm{~K}$. As a consequence of a singlet ground state $A_{1}$, the $\mathrm{Fe}_{\theta}{ }^{4+}$ ion reveals typical Van Vleck-type paramagnetic behaviour [4].

For all $\mathrm{Mn}$-, Co- and Fe-DMS antiferromagnetic coupling between magnetic ions was observed $[5,6]$. In the case of Mn-DMS it was rather well established, both by experimental and theoretical study, that this interaction is dominated by superexchange $[7,8]$. Athough no theoretical model was developed for the exchange interaction between Fe ions, it is expected that also in this case superexchange is the driving force behind the interaction. In view of that the study of chemical trends of exchange for Fe-DMS may be very helptul in formulating a proper model for $d-d$ exchange in these materials. We therefore thought it worthwhile to complete the available data by studying exchange interaction in $\mathrm{Cd}_{1 . x} \mathrm{Fe}_{\mathrm{x}} \mathrm{S}$. In the present work we estimated the nearest neighbour coupling constant from magnetic susceptibility data, using the high temperature expansion series developed for Fe-DMS.

It was shown that susceptibility for high temperatures can be expanded as a Curie-Weiss law [9]:

$$
x=\frac{G(x)}{T-\theta(x)}
$$

where the Curie constant

$$
G(x)=\frac{\mu_{s}{ }^{2} N M_{z}^{2}}{k_{D}} x=C_{0} x
$$

and a Curie-Weiss temperature

$$
\begin{gathered}
\theta(x)=\left(\frac{\left\langle M_{z}^{2}<<B-<M_{z}^{2} B S\right.}{\left\langle M_{z}^{2}>k_{d}\right.}\right)+\left(\frac{2<M_{z} S_{z}^{2}}{\left\langle M_{z}^{2}\right\rangle} \sum z_{p} \frac{J_{p}}{k_{B}}\right) x \\
=A+\theta_{0} x_{D}
\end{gathered}
$$


where $x$ is the concentration of $\mathrm{Fe}$ ions, $<. . .>$ denotes the average value of energy ( $(\boxminus$, magnetic moment (M) and spin (S) operators calculated for isolated (noninteracting) $\mathrm{Fe}$ ion eigenfunctions at infinite temperature. $J_{p}$ is the exchange integral for the $p$-th coordination sphere, $z_{p}$ is the coordination number, $N$ is the number of $\mathrm{Fe}$ ions in $1 \mathrm{~g}$ of CdFeS, $\mu_{\theta}$ is the Bohr magneton and $k_{0}$ is Boltzmann's constant. The above equations were used to estimate the nearest neighbour (NN) interaction for several Fe-DMS $[9,4,10,11]$. We studied hexagonal, single phase $\mathrm{Cd}_{1 . x} \mathrm{Fe}_{\mathrm{x}} \mathrm{S}$ crystals with $x<0.1$. The $\mathrm{Fe}$ concentration in our crystals was determined by atomic absorption on selected pieces, giving values of $0.0081,0.0159$ and 0.087 . In the case of the first two crystals the exact value of $x$ was important for the determination of parameters. Therefore we compared low temperature (4.2K) magnetization of the pieces checked by atomic absorption and those, for which susceptibility was measured. In this way the actual concentration was obtained from the atomic absorption value scaled by the ratio of the magnetization measured at the same magnetic field and temperature. The resulting concentrations were about $10 \%$ lower: $x=0.0071$ and 0.014 .

We measured magnetization (using a SQUID magnetometer) at $2 \mathrm{~T}$ and $5 \mathrm{~T}$ in the temperature range 70-350K. Since in our temperature and field range magnetization is isotropic, the samples were not oriented in respect to the magnetic field. The susceptibility $\chi$ was obtained as $\chi=M / B$. The data were corrected for the diamagnetic susceptibility of the CDS lattice $\left(x_{0}=-3.7 \star 10^{-7}\right.$ emu/g [13]). At the lowest temperatures ( $(<100 \mathrm{~K}$ ) we found that susceptibility determined in $5 T$ is slightly (about 1\%) smaller than that at 2T. This indicates that at 5T and below 100K, we are beyond the linear response limit. For $T>100 \mathrm{~K}$ the difference between $2 T$ and $5 T$ was below experimental accuracy. Nevertheless we based our analysis on susceptibility obtained at $2 \mathrm{~T}$.

In Fig. 1 we display inverse susceptibility as a function

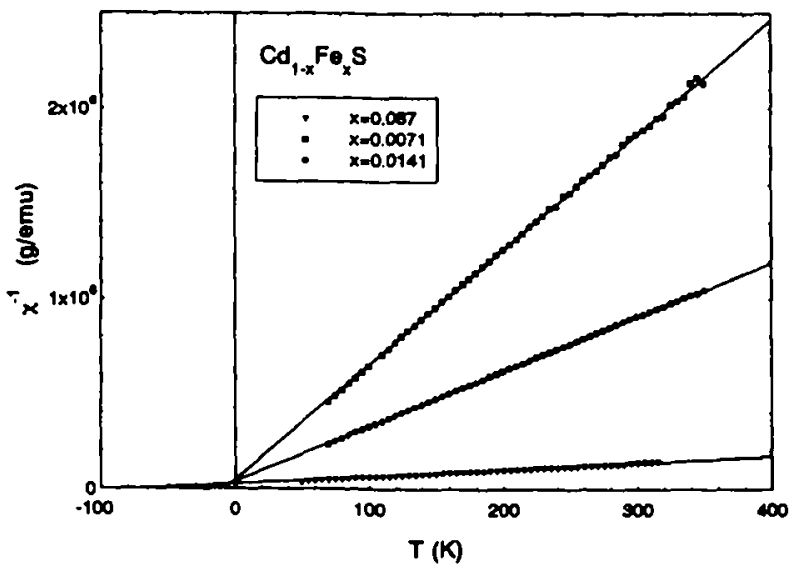

Fig.1 Inverse magnetic susceptibility of $\mathrm{Cd}_{1-x} \mathrm{Fe}_{x} \mathrm{~S}$ as a function of temperature. Straight lines show Curie-Weiss law with parameters given in Tab.1.
Table I

Relevant parameters for $\mathrm{Cd}_{1-x} \mathrm{Fe}_{x} \mathrm{~S}$

\begin{tabular}{|c|c|c|}
\hline$x$ & $\begin{array}{c}C\left(* 10^{-4}\right. \\
\theta \mathrm{mu} / \mathrm{K} / g)\end{array}$ & $\theta(K)$ \\
\hline 0.0071 & $1.66 \pm 0.01$ & $-8 \pm 1$ \\
\hline 0.014 & $3.47 \pm 0.02$ & $-14 \pm 1$ \\
\hline 0.087 & $27.4 \pm 1$ & $-75 \pm 8$ \\
\hline
\end{tabular}

of temperature. A typical Curie-Weiss behaviour is observed above 100K. Negative Curie-Weiss temperatures indicate antiferromagnetic interaction between $\mathrm{Fe}$ ions, similarly as for the other Fe-DMS. Extrapolation of $\chi^{-1}$ versus $T$, in the temperature range 150-350K, gives Curie-Weiss constants and temperatures, which are tabulated in Tab.1. For the $\mathrm{Cd}_{0.0020} \mathrm{Fe}_{0.0071} \mathrm{~S}$ and $\mathrm{Cd}_{0.000} \mathrm{Fe}_{0.014} \mathrm{~S}$ samples the CurieWeiss temperatures are a few times smaller than the temperature range used for the extrapolation of $\Theta$, which justifies our high temperature regime. This is however not the case for $\mathrm{Cd}_{0.013} \mathrm{Fe}_{0.007} \mathrm{~S}$, for which $\theta$ is only 2-4 times smaller than the experimental temperature range. Therefore this crystal was excluded from the analysis which follows.

The exchange parameter $\left(\Sigma z_{,} J_{p}\right)$ can now be determined from the equations given above. We first notice that both $\theta(x)$ and $C(x)$ extrapolate to zero as $x \rightarrow 0$ (Fig.2). A detailed fit yields $\theta_{0}=-(857 \pm 100) K, A=-$ $(1.9 \pm 4) \mathrm{K}$ and $C_{0}=(2.4 \pm 0.2)^{ \pm 1} 0^{-2}$ emuKJg. The obtained value of $C_{0}$ corresponds to $\left\langle M_{2}^{2}\right\rangle=9.28$.
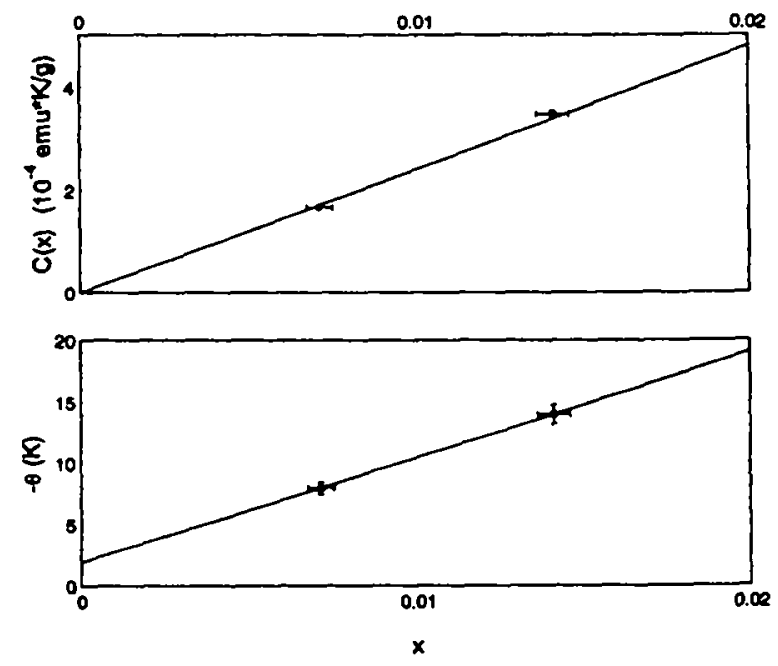

Fig.2 Concentration dependence of the Curie constant $C(x)$ and the Curie-Weiss temperature $\theta(x)$ of $\mathrm{Cd}_{1 . x} \mathrm{Fe}_{\mathrm{x}} \mathrm{S}$. Solid lines represent $\mathrm{C}_{0}=0.0240$ $e m u^{*} K g$ and $\theta(x)=(-1.9-857 x) K$. 
In order to derive the value of $\Sigma z_{p} J_{p}$ we calculated quantum averages $<. . .>$ using $\mathrm{Fe}^{\text {t+ }}$ single ion eigenfunctions, as proposed by Slack at al [3]. For details of calculations we refer to [14]. The eigenstates are parametrized by the crystal field parameter $D q$, parameters describing hexagonality $v, v^{\prime}$ and a spinorbit parameter $\lambda$. Following Ref. 14 we chose: $\mathrm{Dq}=274.8 \mathrm{~cm}^{-1}, \quad v=0, v^{\prime}=35.2 \mathrm{~cm}^{-1}$ and $\lambda=-88.5 \mathrm{~cm}^{-1}$. The summation in the averages $\langle\ldots\rangle$ is performed over ten states of ${ }^{3} \mathrm{E}$ term, since the experimental temperature range is an order of magnitude smaller than the ${ }^{3} \mathrm{E}-{ }^{5} \mathrm{~T}_{2}$ splitting. Therefore our temperature range can be considered to be a high temperature regime in respect to the split the ${ }^{\mathrm{S}} \mathrm{E}$ term, and, at the same time, a low temperature regime relative to the ${ }^{5} \mathrm{E}$ ${ }^{5} T_{2}$ splitting. Detailed calculations of $\chi$ (resulting from the strict diagonalization of an isolated $\mathrm{Fe}$ ion and a Fe-Fe pair - so called Extended Nearest Neighbour Approximation $[4,15])$ shows however that this simplification (i.e. truncation of eigenfunctions basis) yields an overestimation of $\left\langle\mathrm{M}_{2}{ }^{2}\right\rangle$, as well as the parameter $\Sigma z_{p} J_{p}$ of the order of $10 \%$. This effect is not larger than the experimental inaccuracy of $\Theta_{0}$ and was therefore neglected.

We obtained $\left\langle M_{2}^{2}\right\rangle=10.93$ (to be compared to 9.28, the value resulting from the experimental $C_{0}$ value), $\left\langle M_{z} S_{z}\right\rangle=4.24,\left\langle M_{2}{ }^{2} E\right\rangle=-131.9 \lambda^{2} / 10 D q$ and $\langle E\rangle=-11.82$ $\lambda^{2} / 10 \mathrm{Dq}$. We first observe that the resulting $A=+1.00$ $K$ is within the limits of the experimental value of this parameter. Next we find $\Sigma_{p} J_{p}=-(261 \pm 30) K$, which gives the upper estimate for the $N N$ interaction: $\left|J_{N N}\right|<22 \mathrm{~K}$. The actual NN value is lower due to the next neighbours' contribution in the sum $\Sigma z_{p} J_{p}$. A precise determination of this contribution would require information about the interaction range in CdFeS, which is however not available $[4,5,6]$. Assuming the same $J(R)$ dependence for Fe-DMS as was found for Mn-DMS $\left(J(R)=J_{0} R^{n}, \quad n-6.8 \quad[16,5]\right)$, we get $\Sigma z_{p} J_{p}=z_{N w} J_{N W} * 1.09$, and finally $J_{N W}=-(20 \pm 2) \mathrm{K}$.

The obtained $J_{N w}$ is very close to the value for $Z n F e S e$ (Tab.2). We notice that the $\mathrm{ZnSe}$ and CdS host lattices have a very similar NN distance (Tab.2).

As we mentioned above no theory of $d$-d exchange for Fe-DMS has been developed so far, but in principle the same mechanisms which give rise to superexchange for Mn-DMS (i.e. jumping of the valence band electrons to the empty d-orbitals and back) should also be present in Fe-DMS. Moreover, from this point of view, the electronic configurations of the d-level of $\mathrm{Mn}^{+4}$ and

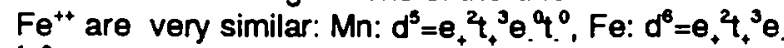
${ }^{\prime} t{ }^{\circ}$, where $t$ and $e$ denote one-electron orbitals of $t$ and e symmetry, subscripts + and - correspond to spin up and down, respectively, and superscripts indicate the occupation of the orbitals. In particular both ions have the same number of empty t-orbitals. We recall that $t$ orbitals hybridize strongly with the valence band and then provide a very effective superexchange channel [18]. If we assume superexchange as the dominant exchange mechanism, then we should expect an
Table II

Nearest neighbour coupling constants and nearest neighbour distances for Fe-DMS

\begin{tabular}{||l|l|c||}
\hline \multicolumn{1}{|c|}{ material } & \multicolumn{1}{|c|}{$J_{\text {NW }}(K)$} & $\begin{array}{c}N N \\
\text { distance }(\mathcal{A})\end{array}$ \\
\hline ZnFeS & $-37[11]$ & 3.83 \\
\hline ZnFeSe & $-22[9]$ & 4.01 \\
\hline CdFeS & -20 this paper & 4.12 \\
\hline CdFeSe & $-19[9.17]$ & 4.28 \\
\hline HgCdFeSe & $-18[17]$ & \\
\hline HgFeSe & $-18[17]$ & 4.30 \\
\hline CdFeTe & $-11[10]$ & 4.58 \\
\hline
\end{tabular}

increasing strength of the exchange with an increase of hybridization, or more precisely, an increase of the matrix element corresponding to the electron transfer between d-level and the valence band, and a decrease of the energy gap between the d-level and the top of the valence band $[7,18]$. On the other hand the matrix element results from the overiap of the wavefunctions and therefore strongly decreases with increasing anion - (magnetic) cation distance. Inspection of Tab.2 shows that such a trend is indeed observed: $\left|J_{N w}\right|$ decreases with increasing NN distance, being the largest for ZnS and the smallest for $\mathrm{CdTe}$. Although a more precise analysis requires information about the location of the d-level relative to the top of the valence band (which is not currently available for Fe-DMS), we notice that the NN distance (and then the matrix element value) seems to be the most important for Fe-DMS. This is particularly well noticeable for the materials with the same anion, where the energy gap between the d-level and the valence band should be roughly cation independent (since the valence band is mostly built of p-type orbitals of the anion). In that sense one could expect that within the group of sulphates (or selenides. tellurides) exchange should vary according to the NN distance, which seems to be the case (cf. Tab.2). However, more pertinent conclusions can only be derived if the superexchange theory for Fe-DMS is elaborated.

Acknowledgments: We acknowledge support from NSC (Taiwan) grant nr. 83-0208-M-002-049, 83-0501-1033-001-A2, 82-0208-M-033-034, 82-0208-M-033-032$T$ and State Committee for Scientific Research (Poland). 
References

1. Semiconductors and Semimetals, 25. "Diluted Magnetic Semiconductors" $\theta$ d. J.K.Furdyna and J.Kossut, Academic Press (1988)

"Diluted Magnetic Semiconductors", ed. M.Balkanski and M.Averous, Plenum Press (1991)

J.Kossut and W.Dobrowolski, in Handbook of Magnetic Materials, ed. K.H.J.Buschow, 7, 231 (1993), North Holland, Amsterdam

2. W.Low and M.Weger, Phys.Rev. 118, 1119 (1960)

3. G.A.Slack, S.Roberts and J.T.Vallin, Phys.Rev. 187, 511 (1969)

4. A.Twardowski, J.Appl. Phys. 67, 5108 (1990), A. Twardowski in "Diluted Magnetic Semiconductors". ed. M.Balkanski and M.Averous, Plenum Press (1991)

5. A. Twardowski, Physica Scripta, T39, 124 (1991)

6. W.J.M de Jonge and H.J.M.Swagten, J. Magnetism and Magn.Mat. 100, 322 (1991)

7. B.E.Larson, K.C.Hass, H.Ehrenreich and A.E.Carisson, Phys.Rev. B37, 4137 (1988)

H.Ehrenreich, K.C.HAss, B.E.Larson and N.F.Johnson, in Materials Research Society Fall Meeting, Boston 1986, Diluted Magnetic (Semimagnetic) Semiconductors, ed. S.von Moinar, R.L.Aggarwal and J.K.Furdyna [Mater. Res. Symp. Proc. 89, 159 (1987)]
8. J.Masek, Solid State Commun., 78, 351 (1991)

9. A.Twardowski, A.Lewicki, M.Arciszewska, W.J.M.de Jonge, H.J.M.Swagten and M.Demianiuk, Phys. Rev. B38, 10749 (1988)

10. M.Arciszewska, A.Mycielski, C.Testelin, C. Rigaux and A.Mauger, Phys. Rev. B45, 8746 (1992)

11. A.Twardowski, T.Fries, Y.Shapira and M.Demianiuk, J. Appl. Phys. 73 (10), 6087 (1993)

12. The more prcise result may be obtained taking into account that at low temperatures only isolated $\mathrm{Fe}$ ions contribute to magnetization. However the resulting correction is of the order of $1 \%$.

13. M.E.Lines and J.V.Waszczak, J. Appl. Phys. 48, 1395 (1977)

14. A.Twardowski, D.Heiman, Y.Shapira, T.Q.Vu, M.Demianiuk, Solid State Commun., 82, 229 (1992)

15. A.Twardowski, H.J.M.Swagten, T.F.H. v.d. Weterig and W.J.M de Jonge, Solid State Commun. 65, 235 (1988)

16. A.Twardowski, H.J.M.Swagten, W.J.M. de Jonge and M.Demianiuk, Phys.Rev. B36, 7013 (1987)

17. A.Twardowski, H.J.M.Swagten and W.J.M.de Jonge, Phys. Rev. B42, 2455 (1990)

18. J.Blinowski and P.Kacman, Phys. Rev. B46, 12298 (1992) 